\title{
Symbolisme et gestion endogène de l'eau en milieu Shabè dans la région Centre du Bénin
}

\author{
Adukê Inuya N. AKPONA ${ }^{12^{\star}}$, Zountchégbé Yves MAGNON², Rigobert C. TOSSOU ${ }^{2}$, Sylvain EFIO² \\ 1 Université de Parakou/ Faculté des Lettres, Arts et Sciences Humaines/Département de Sociologie-Anthropologie. \\ 2 Université d'Abomey-Calavi/ Faculté des Sciences Agronomiques/École d'Économie de Socio-Anthropologie et de \\ Communication pour le développement rural, Laboratoire de Sociologie et de Vulgarisation Rurales. \\ Email : ymagnonfr-yahoo.fr \\ *Auteur correspondant : Adukê Inuya N. AKPONA Email : inuya27@yahoo.fr \\ Original submitted in on $13^{\text {th }}$ November 2015. Published online at www.m.elewa.org on 31st December 2015 \\ http://dx.doi.org/10.4314/jab.v96i1.9
}

\section{RESUME}

Objectif: La problématique de la gestion efficiente des ressources en eau relève d'une préoccupation majeure de nos jours. Cette recherche se propose d'étudier, à partir des représentations sociales de l'eau, les modes endogènes de gestion de la ressource en milieu Shabè au centre du Bénin en vue de contribuer à une gestion efficace et durable de l'eau.

Méthodologie et résultats: L'étude s'est déroulée dans cinq localités de la commune de Savè que sont Djabata, Ouoghi, Kaboua, Diho et Savè-centre. Dans chacune des localités, 40 individus, hommes et femmes, ont été interviewés de manière aléatoire, soit au total 200 individus auxquels se sont ajoutées 4 autorités poilitico-administratives ce qui porte l'échantillon d'étude à 204 individus. La démarche méthodologique adoptée a consisté à l'analyse du contenu des discours autour des évocations liées à l'eau. Les données collectées relatives aux évocations, fonctions et usages de l'eau ont été analysées dans le logiciel SPSS 16. Ainsi le dispositif endogène de gestion de l'eau est un ensemble constitué de règles et interdits liés à l'utilisation de l'eau. Les résultats obtenus montrent que pour $36,45 \%$ des enquêtés, l'eau a une fonction essentiellement religieuse. Les fonctions domestiques et économiques viennent ensuite avec respectivement $35,9 \%$ et $20,75 \%$. L'eau est alors perçue en milieu Shabè, comme une ressource sacrée. Ce caractère sacré, fait que sa gestion constitue un acte religieux dont le déroulement permet la préservation de la ressource. Conclusion et application des résultats: les systèmes modernes de gestion de l'eau et des ressources naturelles en général gagneraient à s'inspirer réellement des modes de régulation endogènes qui demeurent cohérents et efficaces.

Mots-clés: symbolisme, mode de gestion endogène, eau, milieu Shabè, Bénin.

\section{Symbolism and endogenous management of water in Shabè area in central region of Benin}

\section{ABSTRACT}

Objective: The issue of efficient management of water resources is a major concern nowadays. This research intends to study, from the social representations of water, the endogenous modes of resource management in Shabe area in central Benin to contribute to an efficient and sustainable water management. 
Methods and Results: The study took place in five locations in the town that are Djabata Savè, Ouoghi, Kaboua, Diho and Savè center. In each of the localities, 40 individuals, men and women were interviewed at random, a total of 200 individuals which were added 4-poilitico administrative authorities bringing the study sample of 204 individuals. The methodological approach consisted in the content analysis of speeches around the water-related evocations. Data collected on evocations, functions and uses of water were analyzed in SPSS 16. Thus the endogenous system of water management is an established set of rules and prohibitions related to the use of water. The results show that for $36.45 \%$ of respondents, water has an essentially religious function. Domestic and economic functions followed with $35.9 \%$ and $20.75 \%$ respectively. The water is then collected in Shabe area as a sacred resource. This sacred character, that its management is a religious act whose unfolding allows the preservation of the resource.

Conclusion and application of the results: modern water management systems and natural resources in general would benefit from truly inspire endogenous regulatory methods that remain consistent and effective.

Keywords: symbolism, endogenous management mode, water, Shabè area, Benin.

\section{INTRODUCTION}

Les normes qui régissent les différentes sociétés humaines leurs permettent, de s'organiser et de s'auto-gérer dans le temps et dans l'espace (Aberle, 1950). De ce fait, chaque société compte tenu de ses caractéristiques s'organise en fonction d'un certain nombre de modèles en sous-systèmes (Parsons, 1966) ou en systèmes symboliques (Mauss, 2004). Ainsi, dans les sociétés traditionnelles comme la société Shabè, les systèmes symboliques constitués par le langage, les règles matrimoniales, l'art, la science, etc. (Mauss, 2007), contribuent non seulement à leur développement social mais aussi à leur capacité à auto-reproduire leur existence collective (Aberle, 1950). Les hommes et les femmes arrivent ainsi à exercer un contrôle sur leur environnement naturel (Bonte et Izard, 2010) et à gérer de façon efficiente ce que Hardin (1968) appelle les biens communs. Toutefois, la gestion de ces biens communs dépend des rapports particuliers qu'une société entretient avec son environnement (Assaba, 2006). Ainsi pour les peuples agraires comme les Shabè, bien que la

\section{METHODOLOGIE}

Cadre de l'étude : La commune de Savè est située au centre du Bénin dans le Département des Collines à 255 kilomètres au Nord-Ouest de Cotonou, la capitale économique du Bénin. Le choix de cette commune se justifie par le fait qu'elle est l'une des communes les plus peuplées de la région avec une population estimée à 87379 habitants dont 43457 femmes et 43922 hommes (INSAE, 2013). La commune de Savè est traversée par de nombreux cours d'eau qui se déversent dans le fleuve terre soit la première de toutes les ressources naturelles, la nourricière, la pourvoyeuse de richesse, la protectrice et donc la divinité par excellence, on lui associe une autre ressource tout aussi indispensable qui est l'eau. En effet, l'eau joue un très grand rôle dans l'existence et le maintien de tout groupe social. Elle est au cœur de toutes les activités sociale, économique, politique et religieuse et son importance stratégique dans un milieu agraire dépend surtout de son apport dans le quotidien des populations. L'eau est donc aussi en milieu Shabè, " une ressource inestimable ॥ (Assaba, 2006 : p 5758). De ce fait à Savè, ville chef-lieu de l'aire socioculturelle Shabè, l'eau est un lien social et ses modes de gestion et de régulation contribuent à ce que "l'homme transforme de sa main la matière naturelle pour en faire, la maille qui le relie aux siens » (Faïk-Nzuji, 1993 p 24-25). Pour donc être efficace et durable, la gestion de l'eau se fait à travers un certain nombre de symboles que constituent à la fois les interdits, les sanctions et les dons (Mauss, 2007).

Ouémé et dans son affluent l'Okpara. Soit au total un réseau hydrographique long d'environ $147 \mathrm{~km}$ (GabaChabi, 2006). En outre, Savè est dotée d'une station de la Société Nationale des Eaux du Bénin (SONEB) qui s'occupe du traitement et la distribution de l'eau potable. Cependant, l'insuffisance des points d'adduction d'eau potable fait que les populations de cette commune continuent à s'approvisionner au niveau des cours d'eau naturels (okpara). La gestion de ces cours d'eau se fait 
grâce à un dispositif séculaire qui constitue l'objet de cet article.

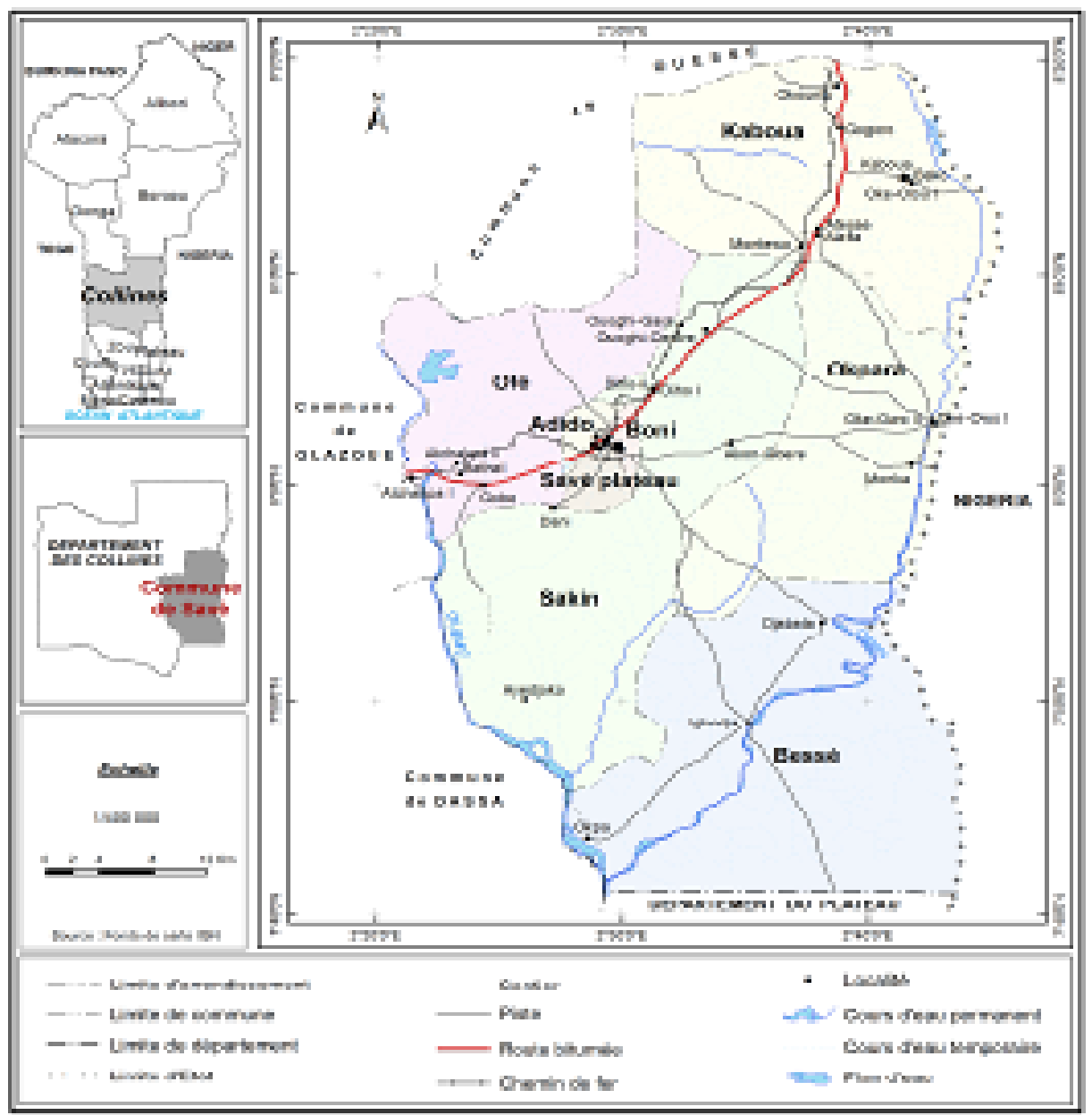

Figure 1: Carte des sources d'eau de Savè Source : Akpona, 2015.

Unités d'observation : Cette recherche a pris en compte les populations de Savè, les autorités politiques et religieuses traditionnelles impliquées dans la gestion de l'eau à savoir: les balè qui sont les chefs de terre, chargés de la gestion et de la régulation foncière, et les agani qui sont les chefs religieux. L'étude s'est déroulée dans cinq localités de la commune de Savè que sont Djabata, Ouoghi, Kaboua, Diho et Savè-centre. Ces localités ont été choisies suivant: le critère de la présence d'au moins une source d'eau naturelle et suivant leur statut, rural ou urbain. Dans chacune des localités, 40 individus, hommes et femmes, ont été interviewés de manière aléatoire, soit au total 200 individus auxquels se sont ajoutées 4 autorités politico-administratives ; ce qui porte l'échantillon d'étude à 204 individus.
Instrument de collecte des données: Les données collectées sont qualitatives et quantitatives et ont été obtenues par de l'observation participante, des entretiens semi-directifs individuels et des questionnaires. L'observation a permis d'appréhender au vif de la réalité les différentes attitudes et postures adoptées par les populations vis-à-vis des sources d'eau. Les entretiens individuels ont ciblé les autorités politico-administratives locales (le Premier Adjoint au Maire, le Directeur des Services Techniques et deux médiateurs sociaux), les autorités traditionnelles (les ministres et conseillers du roi de Savè, les chefs de terre), ainsi que les autorités religieuses. Le questionnaire a ensuite été administré à l'ensemble de l'échantillon d'étude constitué des autorités traditionnelles et religieuses et les populations de Savè. II a permis de recueillir les différentes évocations liées à 
l'eau et les éléments constitutifs du mode endogène de gestion de la ressource dans le milieu. Ces données ont été recueillies à travers quatre (04) catégories d'âges à savoir les moins de vingt ans, les vingt à trente cinq ans, les trente cinq à cinquante ans et les cinquante et plus. Dans la localité, ces tranches d'âge correspondent respectivement aux couches sociales " adolescents », " jeunes », " adultes » et "personnes âgées ». Le choix de ces différentes catégories est motivé par la nécessité d'apprécier l'impact que peuvent avoir les modes endogènes de gestion sur différentes générations.

\section{RÉSULTATS ET ANALYSE}

Différentes évocations ont été recueillies dont les cinq les plus récurrentes sont présentées dans le tableau 1. II ressort de l'analyse du tableau 1 que l'eau suscite des émotions assez contrastées. En effet, pour la majorité des enquêtés (62,3\%), la ressource représente la vie. Elle est une ressource capitale pour les populations de
Traitement et analyse des données : Dans le cadre de cette étude, l'analyse de contenu a été utilisée pour comprendre les logiques et stratégies mises en place par les différents acteurs dans le cadre de la gestion efficace et durable de la ressource hydrique. Cette analyse a pris en compte l'ensemble des représentations sociales associées à l'eau, les modes de gestion et de régulation, les interdits et sanctions liées à son utilisation, les rites propitiatoires destinés à sa préservation.

cette commune. Sa disponibilité apporte au sein des ménages et de la société le bonheur et la joie. L'eau, c'est ce qui sauve et qui unit, et en même temps ce qui tue et qui divise. C'est ce qui explique que les évocations subséquentes telles que la mort les conflits et la maladie.

Tableau 1 : Les cinq premières évocations liées à l'eau

\begin{tabular}{|l|l|l|l|l|l|}
\hline Evocations & Vie & Mort & Conflit & Maladie & Joie \\
\hline Pourcentage & 94,8 & 61 & 25,4 & $22 ; 1$ & 20,5 \\
\hline
\end{tabular}

Source : Enquêtes de terrain, 2015

Ces différentes évocations, donnent de précieuses indications sur le fait que la valeur inestimable qui est accordée à la ressource découle des fonctions qu'elle occupe dans le quotidien des populations de Savè. Ces fonctions sont présentées dans le tableau 2.

Tableau 2: Les fonctions de l'eau selon les populations de Savè

\begin{tabular}{|l|l|l|l|l|l|}
\hline Fonctions & Religieuse & Domestique & Economique & Autres & Total \\
\hline Pourcentages & $36,45 \%$ & $35,9 \%$ & $20,75 \%$ & $6,9 \%$ & $\mathbf{1 0 0 \%}$ \\
\hline
\end{tabular}

Source : Enquêtes de terrain, 2015

Le tableau 2 montre que l'eau occupe plusieurs fonctions dans la vie des populations Shabè à savoir les fonctions religieuse, domestique et économique. En effet, l'eau entre dans tout ce qui concerne la vie sociale à Savè. Au regard du tableau 2, la fonction la plus importante de l'eau en milieu Shabè est la fonction religieuse.

Tableau 3 : Les usages religieux de l'eau selon les populations de Savè

\begin{tabular}{|l|l|l|l|l|l|l|l|}
\hline Religieux & Libations & Rituels & Sacrifices & Intronisation & Baptême/Prière & Autres & Total \\
\hline Pourcentage & 30,4 & 26,5 & 24 & 12,7 & 3,9 & 2,5 & 100 \\
\hline
\end{tabular}

Source : Enquêtes de terrain, 2015

Le tableau 3 révèle que l'eau représente un canal indispensable qui sert à la communication avec les dieux, les ancêtres et tous les esprits qu'ils soient positifs ou négatifs à travers libation, rituels, sacrifice, intronisation, baptême et prière. Pour mieux comprendre les

Tableau 4 : Sources d'approvisionnement en eau évocations, les fonctions et les usages qui sont faits de l'eau à Savè, il importe de connaître les sources d'approvisionnement en eau. Le tableau 4 nous renseigne sur ces sources. 


\section{Akpona et al. J. Appl. Biosci. 2015 Symbolisme et gestion endogène de l'eau en milieu Shabè dans la}

région Centre du Bénin

\begin{tabular}{|l|l|l|l|l|}
\hline \multicolumn{2}{|l|}{ Sources d'eau pour tout usage } & Effectif & Pourcentage & \\
\hline \multirow{3}{*}{ Naturelles } & Marigots & 79 & 38,73 & \\
\cline { 2 - 4 } & Puits & 32 & 15,69 & 54,42 \\
\hline Modernes & Pompes & 93 & 45,58 & 45,58 \\
\hline Total & ---- & 204 & 100 & 100 \\
\hline
\end{tabular}

Source : Enquêtes de terrain, 2015

La lecture des résultats renseigne que $54,42 \%$ des enquêtés s'approvisionnent en eau uniquement au niveau des sources d'eau naturelles (marigots et puits), tandis que $45,58 \%$ d'entre s'approvisionnent au niveau des sources d'eau potable (pompes). Ces résultats donnent deux principales informations à savoir :

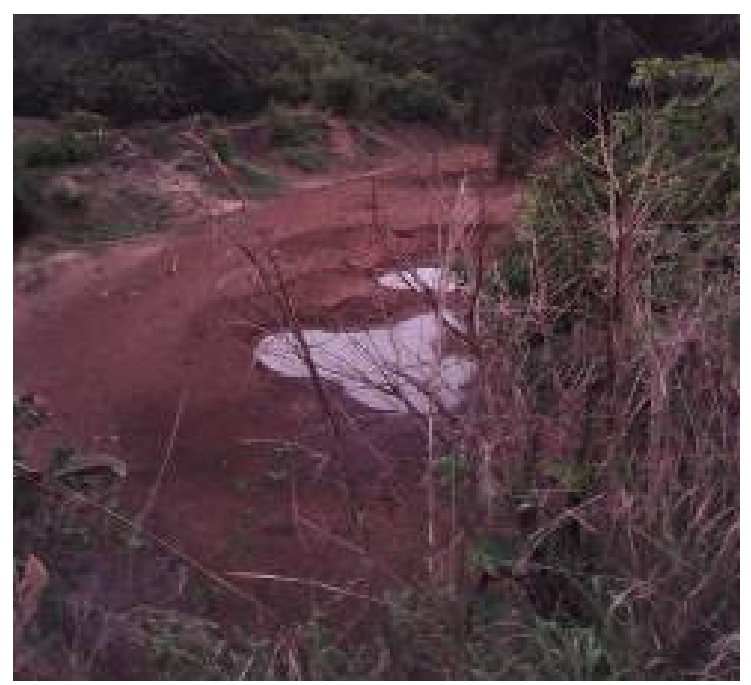

Photo1 : source d'eau tarie à Savè

Source : Akpona, 2015

Les photos 1 et 2 montrent qu'en saison sèche, les sources d'eau naturelles s'assèchent, ce qui entraîne des attroupements au niveau des pompes.

Système de règles et d'interdits liés à l'utilisation de l'eau : Il est essentiellement constitué par un ensemble d'interdits et de sanctions liés à l'utilisation de l'eau. Les interdits sont d'ordre animalier, végétal, astral et chromatique. Ils sont observés au niveau de toutes les sources d'eau naturelles. Les sanctions elles, varient suivant l'importance des infractions commises. Elles peuvent être individuelles ou collectives. La plupart des
- $\quad$ La commune de Savè, manque cruellement de sources d'approvisionnement en eau potable (pompes) ; - $\quad$ La commune de Savè, est sujette en saison sèche à de graves situations de pénuries d'eau. L'incapacité à satisfaire tout le monde en eau entraîne des situations de stress qui débouchent sur des conflits.

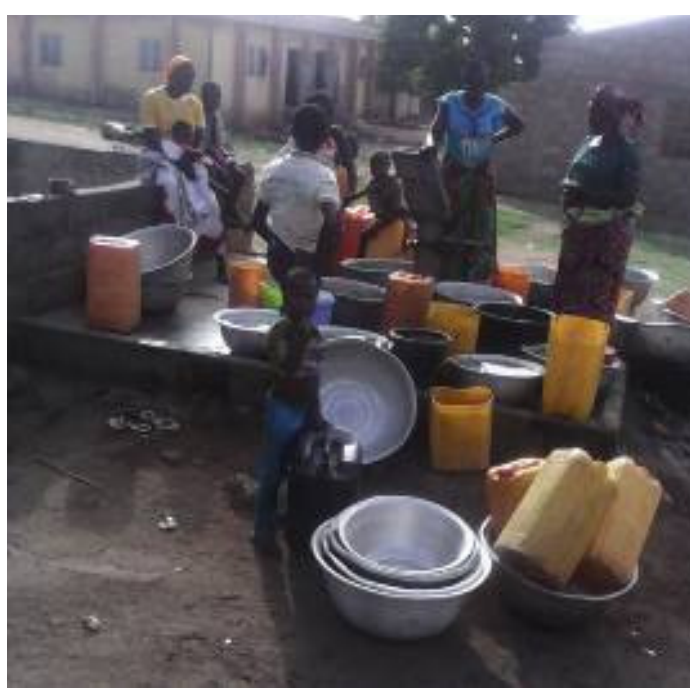

Photo2 : attroupement autour d'une pompe à Savè Source : Akpona, 2015

interdits et sanctions sont communs à toutes les sources d'eau. Cependant, les sources d'eau Okpara et Awouloumi présentent des particularités qu'il importe de relever ici. En effet, ces sources d'eau conservent encore toute la valeur symbolique accordée à l'eau en milieu Shabè.

Interdits liés à Okpara et Awouloumi: Les modes endogènes de gestion de l'eau sont basés sur un certain nombre d'interdits, de sanctions, d'offrandes et de symboles.

Tableau 5 : Les interdits liés à l'usage de Okpara 
Akpona et al. J. Appl. Biosci. 2015 Symbolisme et gestion endogène de l'eau en milieu Shabè dans la région Centre du Bénin

\begin{tabular}{|c|c|c|c|c|c|}
\hline Gestionnaires & $\begin{array}{c}\text { Animal } \\
\text { symbole de } \\
\text { Okpara }\end{array}$ & Interdits & Sanctions & $\begin{array}{l}\text { Offrandes } \\
\text { en cas de } \\
\text { violation }\end{array}$ & $\begin{array}{l}\text { Types de symboles } \\
\text { Identifiés }\end{array}$ \\
\hline \multirow{5}{*}{$\begin{array}{l}\text { - Collectivité } \\
\text { des Omo } \\
\text { Djabata } \\
\text { (homme) } \\
\text { - Adeptes de } \\
\text { la divinité } \\
\text { Oduduwa }\end{array}$} & \multirow{5}{*}{$\begin{array}{l}\text { Serpent et ou } \\
\text { crocodile }\end{array}$} & $\begin{array}{l}\text { Puisage de } \\
\text { l'eau au } \\
\text { zénith ou au } \\
\text { couché du } \\
\text { soleil } \\
\end{array}$ & \multirow[t]{2}{*}{$\begin{array}{c}\text { Individuelle: } \\
\text { piqûres } \\
\text { d'abeilles, } \\
\text { rougeole, perte } \\
\text { de vue (cécité) }\end{array}$} & \multirow[t]{5}{*}{ Coq et poule } & $\begin{array}{l}\text { Astral (soleil) : heure de } \\
\text { puisage de l'eau }\end{array}$ \\
\hline & & le rouge & & & $\begin{array}{c}\text { Chromatique : (le rouge) } \\
\text { interdiction de se rendre au } \\
\text { cours d'eau avec tout objet } \\
\text { rouge }\end{array}$ \\
\hline & & & \multirow{3}{*}{$\begin{array}{l}\text { Collective : } \\
\text { morts subites } \\
\text { par noyade ; } \\
\text { maladies } \\
\text { hydriques }\end{array}$} & & Végétal : Aba, palmier, cola \\
\hline & & Le cochon & & & \multirow{2}{*}{$\begin{array}{l}\text { Animalier : Ogan ou oiseau } \\
\text { gendarme (tisserin), le } \\
\text { serpent, le cochon, le chien, } \\
\text { la poule ou le coq selon le } \\
\text { sexe de l'offrant }\end{array}$} \\
\hline & & Le chien & & & \\
\hline
\end{tabular}

Source : Enquêtes de terrain, 2015

Tableau 6: Les interdits liés à l'usage de Awouloumi

\begin{tabular}{|c|c|c|c|c|c|}
\hline Gestionnaires & $\begin{array}{c}\text { Animal } \\
\text { symbole de } \\
\text { Awouloumi }\end{array}$ & Interdits & Sanctions & $\begin{array}{l}\text { Offrandes } \\
\text { en cas de } \\
\text { violation }\end{array}$ & $\begin{array}{l}\text { Types de symboles } \\
\text { Identifiés }\end{array}$ \\
\hline \multirow[t]{4}{*}{$\begin{array}{l}\text { - collectivité } \\
\text { Omonkin }\end{array}$} & \multirow{4}{*}{ Serpent } & $\begin{array}{l}\text { Puisage de } \\
\text { l'eau au } \\
\text { zénith ou } \\
\text { au couché } \\
\text { du soleil }\end{array}$ & \multirow[t]{2}{*}{$\begin{array}{c}\text { Individuelle : } \\
\text { maladie et } \\
\text { problèmes divers au } \\
\text { quotidien) }\end{array}$} & \multirow{4}{*}{$\begin{array}{l}\text { Chèvre } \\
\text { pour les } \\
\text { femmes, } \\
\text { béliers pour } \\
\text { les } \\
\text { hommes, } \\
\text { cola à } \\
\text { quatre } \\
\text { lobes, coq, } \\
\text { fromage } \\
\text { blanc et } \\
\text { tchakpalo }\end{array}$} & $\begin{array}{l}\text { Astral (soleil) : heure de } \\
\text { puisage de l'eau }\end{array}$ \\
\hline & & \multirow[t]{3}{*}{ le rouge } & & & $\begin{array}{c}\text { Chromatique : (le rouge) } \\
\text { interdiction de se rendre au } \\
\text { cours d'eau avec tout objet } \\
\text { rouge et le blanc (symbole de } \\
\text { pureté) }\end{array}$ \\
\hline & & & \multirow[b]{2}{*}{$\begin{array}{l}\text { Collective : morts } \\
\text { subites par noyade } \\
\text { les maladies } \\
\text { hydriques en } \\
\text { particulier au sein de } \\
\text { la collectivité des } \\
\text { Omonkin }\end{array}$} & & Végétal : mil (tchakpalo), cola \\
\hline & & & & & $\begin{array}{l}\text { Animalier : le serpent, le coq, } \\
\text { le bélier ou la chèvre selon le } \\
\text { sexe de l'offrant }\end{array}$ \\
\hline
\end{tabular}

Source : Enquêtes de terrain, 2015

A la lecture des tableaux 5 et 6 on remarque que les modes endogènes de gestion de l'eau sont basés sur un certain nombre de symboles que l'on peut regrouper en quatre grandes catégories à savoir les symbolismes animalier, végétal, astral et chromatique. Le symbolisme, un mode très efficace de gestion de l'eau en région Shabè
Le symbolisme animalier : Plusieurs espèces du règne animal de part leur signification dans la culture Shabè participent à la bonne gestion de l'eau. Au nombre de ces animaux on peut citer : le serpent, le poulet et le bélier.

" ejo » ou le serpent : Il symbolise pour les Shabè l'eau. Le serpent représente l'équilibre social et écologique. II 
est révélateur des problèmes liés à l'usage de l'eau ce qui fait qu'il est craint et inspire aux populations de cette commune un profond respect. En effet, son apparition dans l'eau ou aux abords d'une source d'eau est symbole d'une infraction à l'un des interdits liés à l'utilisation de la ressource. Pour éviter donc que la colère de la divinité n'emporte tout le village, des animaux tels que le poulet (coq ou poule) et le bélier lui sont offerts pour l'apaiser.

"Adiè », le poulet : En milieu Shabè, le poulet est un animal social par excellence. II symbolise l'accueil et la reconnaissance. Son offrande est supposée consolider les liens affectifs et spirituels entre celui qui l'offre et celui qui le reçoit. C'est pourquoi le coq ou la poule est l'animal de prédilection de tous les rites destinés à apaiser la divinité.

"Aako ", le bélier : Le bélier chez les Shabè est doté d'une grande charge symbolique. II représente, la force de la nature. Le bélier notamment celui à pelage blanc, est un animal majestueux qui symbolise l'image de la puissance et surtout de l'abondance et de la richesse. Selon les enquêtés, c'est le don le plus précieux que l'homme peut offrir pour honorer l'eau. C'est un animal sacrificiel très important dont la valeur est inestimable. En plus de sa valeur sacrificielle, son coût dissuade les usagers de toute transgression des normes à travers le respect des préceptes symboliques liés à l'utilisation de l'eau

Le symbolisme végétal : Tout comme les animaux, les végétaux jouent un rôle important dans la gestion endogène de l'eau. Parmi les végétaux caractéristiques de l'eau on a : ợpe et $A b a$.

" ope » ou Elaeis guinensis (Jacq.) : Communément appelé ope, le palmier joue un très grand rôle dans la protection des sources d'eau naturelles. Le palmier sertà protéger l'écosystème aquatique à travers la régulation de son exploitation par les populations. En effet, la présence des rameaux de palmier dans une source d'eau est un indicateur de la présence des dieux donc du caractère sacré de cette partie interdite d'exploitation.

" Aba » ou le Bauhinia thonningii (Schum.) : C'est un arbre très important dans l'organisation de la gestion endogène de l'eau. En effet, sa prolifération dans un endroit, indique la présence toute proche de la nappe phréatique. II permet aux autorités traditionnelles de gestion de l'eau d'identifier les probables lieux de forages. Aba permet également de purifier l'eau de par sa présence.

Le symbolisme astral : Deux principaux astres entrent en ligne de compte dans la gestion endogène de l'eau chez les Shabè : le soleil et la lune. Ces astres sont considérés comme étant les maîtres du temps.
" ohun " ou le soleil: II symbolise la positivité, la lumière et la vie. Sa position dans la journée détermine les heures de fréquentation et de puisage de l'eau. Ainsi de son lever vers $5 \mathrm{~h}$ du matin à son zénith $12 \mathrm{~h}-13 \mathrm{~h}$, les sources naturelles sont " ouvertes» au puisage. Elles sont interdites à l'exploitation humaine de $13 \mathrm{~h}$ à $16 \mathrm{~h}$, moment où les dieux sont supposés s'abreuver. Ensuite les sources sont réouvertes aux humains jusqu' à 20h, heure à laquelle les dieux sont supposés reprendre à nouveau le contrôle. Le soleil est considéré comme le régulateur naturel du puisage de l'eau des puits, marigots, rivières, fleuves etc..

" oshukpa » ou la lune: Pendant nocturne du soleil, la lune symbolise le pouvoir des dieux et celui des "lya " c'est-à-dire des « mères ». Elle préside le puisage rituel de l'eau qui participe aux rites de propitiation dus au mésusage de l'eau. Puiser l'eau en pleine nuit, sert à confier à la lune toute la négativité et tous les problèmes du village. Le puisage ici est fait par des initiés.

Le symbolisme chromatique: Le symbolisme chromatique est très important dans l'organisation de la gestion endogène de l'eau en milieu Shabè. En effet, ce symbolisme est en corrélation directe avec la qualité de l'eau. L'eau est supposée être de couleur blanche « Omi, n'kin founfoun ni » (l'eau est de couleur blanche). Pour le Shabè, le blanc symbolise la pureté, l'innocence, la richesse et la paix. C'est la couleur de Oxala, ou Obatala qui est la divinité purificatrice de l'eau. Aussi lorsque les Shabè offrent aux dieux de l'eau un bélier, un coq ou une poule, des colas et du fromage blancs elles sont supposées, par ce geste purifier l'eau et lui redonner toute la charge symbolique qui à un moment donné a été atténuée par des mésusages. Ces mésusages se traduisent dans le milieu par une infraction qui est lourde de conséquences: l'apport de tout objet (bassine, vêtements, etc.) de couleur rouge aux abords des sources d'eau. Le rouge symbolise la saleté et l'impureté car associée par les Shabè à la période néfaste du mois que constituent les menstruations de la femme. Ainsi, lorsque vous amenez le rouge à une source d'eau (rivière, marigot, barrage, puits et pompes), vous êtes censés souiller cet élément vital et pur en le transformant en vecteur de maladies donc en un agent de mort. Afin de respecter cet interdit, les animaux offerts aux divinités de l'eau en cas d'infraction ne sont pas immolés mais relâchés à des endroits indiqués par la présence des rameaux de palmier. Ces précautions sont prises afin d'éviter que le sang (élément de couleur rouge) ne coule au bord des sources d'eau. En effet, le rouge est supposé perturber le sommeil des dieux de l'eau, stimuler leur agressivité et réveiller en eux le côté obscur de leurs 
identités. L'apport du rouge devient destructeur tant pour les dieux que pour les hommes.

Acteurs de la gestion et de la préservation de l'eau : Ils sont de deux ordres : nouménale et phénoménale. En effet, la gestion endogène de l'eau relève à la fois des dieux et des hommes. Des dieux parce qu'ils sont à la fois donateurs et gardiens de la ressource et des hommes parce qu'ils sont usagers et gestionnaires de l'eau.
Acteurs du monde nouménal : Ils sont constitués des divinités. Les divinités impliquées dans la gestion de l'eau en milieu Shabè sont nombreuses et variées. Elles incarnent toutes les catégories de la ressource, allant des eaux salées aux eaux souterraines en passant par les eaux douces. Les acteurs du monde nouménal peuvent être regroupés en deux grandes catégories : les maîtresordonnateurs et les auxiliaires-exécuteurs tel que le montre le tableau 7.

Tableau 7 : Récapitulatif des acteurs du monde nouménal

\begin{tabular}{|l|l|l|}
\hline Groupe & Divinités & Rôles \\
\hline \multirow{4}{*}{ Maîtres-ordonnateurs } & Yemadja, & Pourvoyeuse d'eau et de fertilité \\
\cline { 2 - 3 } & Shankpanan & Justicière, chargée d'appliquer les sanctions \\
\cline { 2 - 3 } & Oxala ou Obatala. & Purificateur de l'eau \\
\cline { 2 - 3 } & Ifa & Juge de paix \\
\hline \multirow{2}{*}{ Auxiliaires-exécuteurs } & Okpara & Pourvoyeurs et justiciers \\
\cline { 2 - 3 } & Awouloumi. & \\
\hline
\end{tabular}

Source : Enquêtes de terrain, 2015

Les acteurs du monde phénoménal : Trois catégories d'acteurs sont impliquées dans la gestion de l'eau. II s'agit des politiques, des religieux et des populations de Savè. La catégorie des politiques est constituée par le roi qui est chargé en collaboration avec ses ministres et les chefs terre (balè) de faire respecter les règles liées à l'utilisation de l'eau. Ils assurent également une égale répartition de la ressource à travers tout le royaume en sacralisant des réceptacles naturels de l'eau que sont les crevasses au flanc des collines, les trous d'eau. La catégorie des religieux est celle formée par les Agani, les chefs religieux. Ils sont les porte- voix des dieux auprès des hommes. Ces chefs religieux sont chargés d'édicter les règles d'usage et de gestion de l'eau et de les actualiser. Cette catégorie d'acteurs a également en

\section{DISCUSSION DES RÉSULTATS}

La gestion endogène de l'eau en milieu Shabè est un système de corrélation entre la culture du groupe et chacune des individualités qui le composent (LéviStrauss, 2012). Ainsi, la représentation de l'eau comme ressource sacrée modèle et influence les comportements individuels vis-à-vis de la ressource. L'eau est une réalité concrète parce que la société lui a conféré non seulement un nom Omi, (Blot, 2004), mais un visage, Ejo, une odeur et un goût. Elle a une double existence : phénoménale et nouménale. Une existence phénoménale parce que l'eau relève du domaine des hommes, elle participe à la vie sociale de ce peuple. L'eau est garante de la survie et la cohésion sociale à Savè grâce aux fonctions domestique, religieuse, économique et politique qu'elle assure. Cette charge d'identifier les nouvelles sources susceptibles d'être sacralisées, d'invoquer les dieux de l'eau et d'écouter leurs plaintes et doléances. Les chefs religieux se chargent ensuite de transmettre ces plaintes et doléances des divinités aux politiques et aux populations et établissent avec eux, un commerce au sens maussien du terme, fait d'échanges symboliques : offrandes contre abondance de l'eau de bonne qualité (Mauss, 2007 : 7778). Les populations quant à elles occupent une place importante dans le dispositif de gestion endogène de l'eau. C'est en effet à elles qu'échoit, le respect des normes et interdits prescrits par les dieux et les autorités traditionnelles. Et c'est d'ailleurs en raison de leur perméabilité à ce système symbolique de normes que perdurent ces modes endogènes de gestion de l'eau.

importance sociale de l'eau lui confère un caractère sacré qui détermine son existence nouménale. L'eau est alors une ressource sacrée parce qu'elle est socialement définie ou nommée (Berger et Luckmann, 1996). Toutefois, en insistant sur la dualité de l'eau (nouménale et phénoménale) il ne s'agit pas d'instaurer un débat entre la primauté de l'institutionnel sur le culturel ou viceversa, mais plutôt de mettre l'accent sur le fait que c'est de la nature même de la société de s'exprimer symboliquement à travers ses coutumes et dans ses institutions (Lévi-Strauss, 2012). C'est donc en raison de ces échanges symboliques que les transgressions sociales à l'usage de l'eau sont considérées comme des conduites anormales qui créent un déséquilibre social qui 
se manifeste par les maladies, la sècheresse, les noyades, les conflits. La correction de ce déséquilibre se fait en entrant en commerce avec les dieux de l'eau à travers les offrandes et les sacrifices (Mauss, 2007 : 77 78). Ce commerce bien élaboré prend en compte un ensemble de symboles aussi bien animal, végétal, astral que chromatique qui constituent des liens utiles (FaïkNzuji, 1993) entre les hommes et les dieux. Mais, ce qui interpelle ici est moins le déroulement des rites de propitiation que leur existence. En effet, bien qu'étant souvent individuels, les transgressions sociales ou comportements anormaux relatifs à l'eau, traduisent néanmoins une défiance du groupe à l'autorité, qu'elle soit suprême à l'instar des dieux ou intermédiaire comme les autorités traditionnelles. II est important de rappeler ici, la corrélation entre comportements individuels et culture. De fait, c'est l'existence même des rituels de propitiation qui instaure les comportements anormaux ou mésusages de l'eau. Le mésusage devient dans cet ordre d'idées une nécessité. Elle donne aux différents gestionnaires de l'eau (dieux et autorités traditionnelles) une légitimité et leur confère, un pouvoir de coercition. La société tient alors en tant que système symbolique de ces transgressions sociales. II en ressort que l'efficacité des modes endogènes de gestion de l'eau et leur pouvoir

\section{CONCLUSION}

La gestion endogène de l'eau en milieu Shabè est un système symbolique bien élaboré qui prend en compte d'une part, la diversité des représentations et des pratiques liées à l'eau et d'autre part, les acteurs et les enjeux qui se cristallisent autour de la ressource. Le cadre institutionnel et le système symbolique de gestion endogène de l'eau mis en place, montrent que dans les

\section{RÉFÉRENCES BIBLIOGRAPHIQUES}

Aberle DF ,1950. The functional prerequisites of a society. In: Ethics $60: 100-111$.

Abric J-C, 2001. Les représentations sociales: aspects théoriques. In: Abric J.-C. Pratiques sociales et représentations. Paris, PUF: 11-35.

Abric J-C, 2003. Méthodes d'études des représentations sociales ; Ramonville St Agne, Érès : 295 p.

Assaba C, 2006. Cours d'Anthropologie de la Communication. Université d'Abomey-Calavi, 91p.

Bardin L, 1991. L'analyse de contenu. Paris, Presses Universitaires de France. Page)

Berger R., Luckmann T, 1996. La construction sociale de la réalité. Paris, Armand Colin. Page) coercitif sont plus dus à l'attitude que la société adopte à l'égard de la catégorie d'acteurs que constituent les autorités de gestion qu'à leurs vertus magiques et leurs caractères physiques (Lévi-Strauss, 2012). La gestion endogène de l'eau à Savè peut être appréhendée comme le produit d'une relation où l'eau devient réelle par des pratiques sociales construites collectivement qui lui confèrent non seulement une dimension instrumentale et politique (Blot, 2004), mais surtout une dimension religieuse et symbolique. La gestion et la régulation endogènes de l'eau à Savè est alors un acte éminemment sacré qui renouvelle un pacte entre les hommes et les dieux (Eliade, 2009). Toutefois, aucun système ne peut vivre en autarcie. En effet, toutes les sociétés se développent dans le temps et dans l'espace. De ce fait, une société est sujette à l'influence d'autres sociétés et aux états antérieurs de son propre développement (Lévi-Strauss, 2012). Ainsi, la société Shabè ne peut pas être intégralement et complètement symbolique et c'est sans doute cette « perméabilité » qui explique l'abandon de certaines sources sacrées tombées en latence. En effet, le contact avec d'autres peuples, cultures et religions peut expliquer la recrudescence des mésusages ou comportements anormaux liés à l'usage de l'eau.

sociétés dites « traditionnelles », rien n'est fait au hasard. Le dispositif de gestion mis en place prend en compte l'eau dans sa globalité et dans toutes ses dimensions, physique et symbolique. Ainsi, quel que soit le contexte social, les systèmes modernes de gestion gagneraient à s'inspirer des modes endogènes de gestion de l'eau.

Blanchet A., Gotman A, 2012. L'enquête et ses méthodes : l'entretien. Paris, Armand Colin, 126p.

Blot $F, 2004$. Les rapports entre société et eau: territorialité et/ ou technicité ? In : L'eau à la rencontre des territoires, Montpellier, France. pp.1-3.

Bonte P., Izard M, 2010. Dictionnaire de l'Ethnologie et de l'Anthropologie. Paris, Presses Universitaires de France, 842p)

Capo-Chichi YJ, 2006. Monographie de la commune de Savè, Mission de Décentralisation. In: Programme d'appui au Démarrage des communes, 31p.

Collin J, 2004. L'eau, le miracle oublié. Paris Editions Guy Trédaniel, 237p. 

région Centre du Bénin

Copans J, 2011. L'enquête et ses méthodes. Paris, Armand Colin, 127p.

Durkheim E, 1898. Représentations individuelles et représentations Collectives. In: Revue de métaphysique et de morale. Page)

Durkheim $E$, 2008. Les formes élémentaires de la vie religieuse. Paris, Presse Universitaire de France.

Eliade M, 2009. Le mythe de l'éternel retour. Paris, Gallimard, 158p.

Fairhead J et Leach M, 1992. Représentations culturelles africaines et gestion de l'environnement. In: Science Research Council,14 $p$.

Gaba-Chabi S, 2006. La pénurie d'eau au Bénin : quel avenir pour les femmes et les enfants ? In : Revue ONG Ecolo-Bénin, Bruxelles, 321p.

Hass V, 2006. Les savoirs du quotidien. Transmissions, Appropriations, Représentations. In : Collection: Didact Psychologie sociale, Rennes Les Presses universitaires de Rennes, 274 pp.

Lepage L, 1999. « Les controverses environnementales sont plutôt culturelles que scientifiques». In: Dumas, B., Raymond, C. et Vaillancourt J-C., Les sciences sociales de l'environnement: Analyses et pratiques, Les presses de l'Université de Montréal p. 133-147

Lévi-Strauss C, 2012. Introduction à l'œuvre de Marcel Mauss. Paris Quadrige/Presses Universitaires de France, Nouvelle Edition, revue et corrigée. $56 \mathrm{p}$.

M.Faïk-Nzuji C, 1993. La Puissance du Sacré. Paris, Maisonneuve et Larose, $184 p$.

Mauss M, 2007. Sociologie et Anthropologie : Dons échangés et l'obligation de le rendre. Paris Quadrige/Presses Universitaires de France, Nouvelle Edition, revue et corrigée, $482 \mathrm{p}$.

Vaillancourt J-G, 2001. Religion, Ecologie et Environnement. Université de Montréal. Page)

Verger FP, 1995. Dieux d'Afrique. Paris, Paul Hartman, $416 p$. 